\title{
Acuros-Based Planning with Density Override for Lung SBRT by a Dynamic Conformal Arc Technique: Comparative Evaluation with AAA-Based Planning in Four-Dimensional Dose
}

\author{
Inhwan Yeo*, Neil Joyce, Deepinder P. Singh, Michael T. Milano, Yuhchyau Chen, \\ Sanjukta Bandyopadhyay, Hongmei Yang, Douglas Rosenzweig \\ Radiation Oncology, University of Rochester, Rochester, USA \\ Email: ^medicphys@hotmail.com
}

How to cite this paper: Yeo, I., Joyce, N., Singh, D.P., Milano, M.T., Chen, Y., Bandyopadhyay, S., Yang, H.M. and Rosenzweig, D. (2021) Acuros-Based Planning with Density Override for Lung SBRT by a Dynamic Conformal Arc Technique: Comparative Evaluation with AAA-Based Planning in Four-Dimensional Dose. International Journal of Medical Physics, Clinical Engineering and Radiation Oncology, 10, 94-110.

https://doi.org/10.4236/ijmpcero.2021.1020 $\underline{09}$

Received: March 16, 2021

Accepted: May 28, 2021

Published: May 31, 2021

Copyright $\odot 2021$ by author(s) and Scientific Research Publishing Inc. This work is licensed under the Creative Commons Attribution International License (CC BY 4.0).

http://creativecommons.org/licenses/by/4.0/ (c) (i) Open Access

\begin{abstract}
The purpose of this study was to evaluate a planning strategy based on Acuros with density override in comparison with AAA without and with the override. Ten lung-tumor patients were selected with each PTV size around 2 $-4 \mathrm{~cm}$ and were imaged using slow scan, followed by four-dimensional (4D) imaging limited to the target. On each phase-specific image, gross tumor volume (GTV) was contoured. Summed over all phases, an integrated GTV (iGTV) was generated and copied to the slow scan. A treatment plan was created using a dynamic-conformal-arc technique with AAA to prescribe 60 Gy to $95 \%$ of PTV (iGTV $+0.5 \mathrm{~cm}$ ). Each AAA-based plan was regenerated by overriding the density of the setup margin of PTV by GTV density (modeling tumor-position uncertainty). It was also regenerated with Acuros and the override. The three plans were validated in $4 \mathrm{D}$ dose to PTV, after similarly overriding PTV density (phase-specific), accurately calculating with Acuros, and summing the phase-specific plans through organ/dose registration. The Acuros-based plan with the override, the AAA-based plan, and the AAAbased plan with the override provided 4D PTV doses of 63.9, 67.9, and 62 Gy at $\mathrm{D} 95 \%$, respectively, averaged over all patients. The override with Acuros and AAA produced lesser $4 \mathrm{D}$ doses, closer to the associated 3D doses, respectively, than that without the override, with better conformity and inhomogeneity. With the override in common, Acuros provided a greater dose to PTV than that by AAA. The Acuros with the override, which was more accurate than the AAA without the override, is clinically recommended.
\end{abstract}

\section{Keywords}

Lung SBRT, Acuros, Density Override, AAA, 4D Dose Calculation 


\section{Introduction}

Stereotactic-body-radiation therapy (SBRT) has been widely used for the treatment of relatively small lung tumors [1]. SBRT is intended to optimize tumor control while maximally sparing normal tissues such as the lung. To achieve this goal, it is delivered in a small number of treatment fractions by providing a highly conformal dose around the tumors [2]. A recognized challenge with lung SBRT is dose calculation accuracy in the scenario of two interfaced materials of lung and small tumors [3] [4]. Due to the sharp density change in the interface and the insufficient distance of dose buildup and lateral radiation transport in the tumors, this condition challenges accurate dose calculation in the tumors and interface areas. This challenge is further complicated as the setup margin of the tumors is occupied by the lung that cannot represent them in terms of a similar physical density.

Traditionally, like others, our institution has used algorithms such as analytic anisotropic algorithm (AAA) that is based on predetermined dose deposition kernels and is relatively accurate for nominal dose calculations other than those in highly inhomogeneous media [3]-[12]. Since the introduction of a deterministic-radiation-transport algorithm, called Acuros XB (Acuros), for dose calculation in radiation therapy, its accuracy has been extensively evaluated in computer tomographic (CT) images of patients [3] [5]-[11] as well as phantoms [4] [12] [13]. Evaluations using conformal beams, not involving intensity modulation, have offered an understanding of the fundamental performance of Acuros, unaffected by the performance of beam optimization [4] [7] [8] [9]. Investigators have found that Acuros depicts a more inhomogeneous dose to the tumors, reflecting a greater accuracy of dose calculation, compared with AAA dose, that was comparable to phantom measurements [4] and Monte Carlo dose calculations [13].

At our institution, for more accurate dose calculation using conformal beams we have planned to implement Acuros for lung SBRT through realistic modeling of the setup margin of planning target volume (PTV) that are defined for iGTV. During treatment, the geometrical area of the margin may be occupied by iGTV due to typical setup uncertainties, but this area is fully occupied by lung tissue during planning [14]. This discrepancy, due to the difference between tissue densities of tumor and lung, is associated with a difference between radiation transport characteristics in lung and iGTV. Due to this difference, therefore, the calculated dose in PTV may not represent that in iGTV, a situation that is clinically undesirable. Similar to the uncertainty of the setup margin, the area of the iGTV is in part occupied by the moving tumor and in part by lung tissues at any given time, while it is fully occupied by the tumor during planning (via averaged tumor density over all phases). This may cause the dose calculated in the iGTV to inaccurately model the dose in GTV. The above limitations in modeling the setup uncertainty and the tumor motion will be more accurately realized when Acuros is used as it provides more physically correct modeling of radiation 
transport than AAA does [4] [7] [8] [9] [13]. In a study by Wiant et al. [14], the PTV margin and the area of the internal target volume (ITV) during planning was density-overridden with the tumor. This was to accurately model dose to GTV that moves during treatment. The override caused better agreement with measurements of delivered dose in a phantom, than the plans with no override performed when AAA was used. In this study, we have proposed the override similarly when Acuros is used with the intent of providing the more geometrically and physically accurate dose calculation in the targets. We have comparatively evaluated the proposed plan with the classic AAA-based plan without the override (past practice) in terms of four-dimensional (4D [15]) doses planned in GTV and its PTV (PTV_4D) as well as the three-dimensional (3D) doses in iGTV and PTV, while the 4D doses can serve as benchmarks for the 3D doses. Our intent was to evaluate the proposed planning strategy in comparison with the past strategy for its clinical use. However, to evaluate the proposed plan on equal conditions with the classic AAA plans, we have imposed the override to the latter as well and comparatively evaluated. The $4 \mathrm{D}$ dose [15] was utilized in addition to the 3D dose in order to consider the temporal nature of the area of iGTV. The reported greater dose inhomogeneity associated with Acuros [4] [7] [8] [9] could lead to greater MUs than those associated with AAA. We were interested in evaluating the impact of the override on MUs, and the $4 \mathrm{D}$ and $3 \mathrm{D}$ doses to the targets.

\section{Methods}

\section{1) Patient Selection}

This study was approved by the University of Rochester Medical Center Human Subjects Research Board (Internal Review board). Ten patients who had been treated with SBRT to their lungs using dynamic conformal arc (DCA) between 2013 and 2016 were selected based on the following criteria: 1) tumors were separated from the chest wall or mediastinum by greater than $1.5 \mathrm{~cm}$ (equating to PTV being separated by at least $1 \mathrm{~cm}$ ); 2) tumor sizes were between $2-4 \mathrm{~cm}$ in either their lateral or longitudinal dimensions; and 3) treatment plans had been generated using $6 \mathrm{MV}$ X-rays and AAA for a total dose of $60 \mathrm{~Gy}$ in 5 fractions, prescribed to $95 \%$ volume of PTV. The first two criteria were used because the performance of Acuros is greatly affected by build-up and lateral scatter conditions [4] [13].

2) Simulation and 3D Treatment Planning

During simulation, patients with free breathing underwent slow CT followed by limited-length $4 \mathrm{D}$ CT to the region of the PTV, as per departmental imaging protocols; the former was used for planning and the latter was used for tumor delineation. Three standard DCA beam orientations that had been used are defined by the following rotational gantry and fixed couch angles for tumors positioned in the left lung of patients: from $340^{\circ}$ through $179^{\circ}$ with $0^{\circ}$; from $0^{\circ}$ through $80^{\circ}$ with $350^{\circ}$; from $100^{\circ}$ through $170^{\circ}$ with $10^{\circ}$ with the exception of one patient whose arm could not be raised (see Figure 1). No lateral margin 


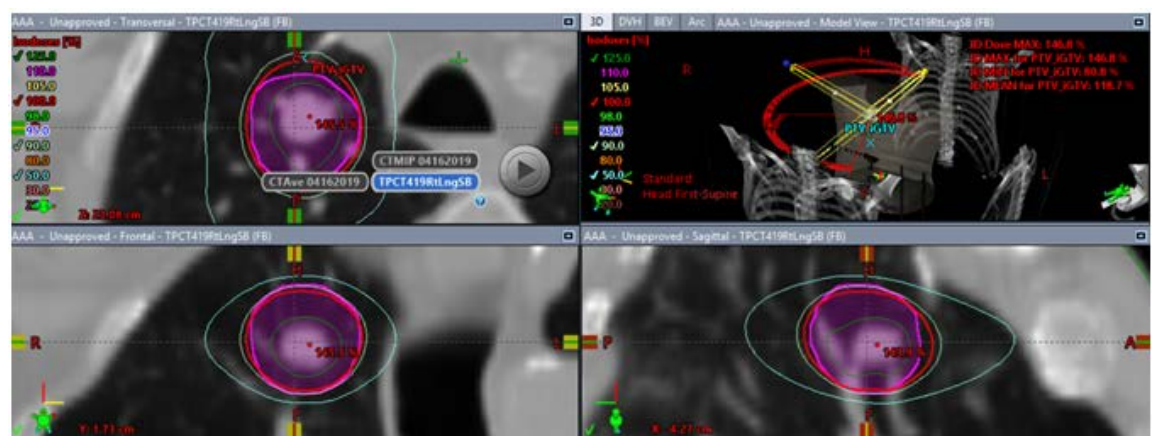

Figure 1. Treatment plan by conformal arcs. Top-left: Axial plane; Bottom-left: Coronal plane; Top-right: $3 \mathrm{D}$ view. The purple contour is PTV.

between PTV and the aperture was used, but superiorly and inferiorly $1 \mathrm{~mm}$ was used. The gantry and couch angles for tumors positioned in the right lung are symmetric with respect to the anterior-to-posterior line. This approach (DCA as opposed to VMAT or IMRT) was preferred for many patients whose DCA plans met institutional dosimetric constraints, as the delivery time is relatively fast $(<2$ minutes) in part assisted by the auto setup of gantry and couch, so that the possibility of respiratory baseline shifts is minimized [16]. The constrains were V20 Gy $<10 \%$ for total lung minus GTV, $\leq 30$ Gy max for spinal cord, $\leq 40 \mathrm{~Gy}$ max for esophagus, and V30 Gy $\leq 30 \mathrm{~mL}$ for chest wall (CW) [17]. They were practically relevant and used across all patients at our institution.

For the intended planning evaluation, the 3D planning based on AAA (calculating dose to water) was performed as follows. GTV in each phase-specific image of 4 DCT images was contoured by our physician authors, and iGTV as an integration of GTV over all phases was generated for each patient. A setup margin of $0.5 \mathrm{~cm}$ was added to iGTV to generate the PTV (PTV_3D). The average PTV volumes across all patients were $15.6 \pm 5.2 \mathrm{~cm}^{3}$ with $5.4 \mathrm{~cm}^{3}$ as the minimum (for patient \#8 or P8) and $23.2 \mathrm{~cm}^{3}$ as the maximum, as shown in Table 1 . The PTV was copied to the slow CT images for each patient because the 4DCT images and the slow CT images were naturally registered to each other. The three DCA beams with equal weighting among them were applied to PTV to generate 3D plans based on AAA (AAA_3D). When the above normalization (\#3 criterion) was used, the constraints were fully met for all ten patients. Although as a part of our clinical practice ITV had been delineated on maximum-intensityprojection images of the 4DCT images, for the calculation of dose on moving tumors (i.e. 4D dose) the above iGTV was newly generated.

The proposed 3D planning based on Acuros (Acuros_DO_3D) was performed by changing AAA with Acuros (calculating dose to medium) and adopting the density override to model the setup uncertainty of iGTV as follows. The mean HU of the iGTV from the MIP image was used to override the density of the area of the setup margin of PTV. The average density of iGTV across all patients was $0.72 \pm 0.09 \mathrm{~g} / \mathrm{cm}^{3}$ with $0.57 \mathrm{~g} / \mathrm{cm}^{3}$ as the minimum and $0.85 \mathrm{~g} / \mathrm{cm}^{3}$ as the maximum, as shown in Table 2. Similarly, adopting the same override and using 
Table 1. Tumor volume $\left(\mathrm{cm}^{3}\right)$ of each patient. V_PTV_3D is the volume of PTV_3D. V_PTV_4D is similarly defined. P1 is the $1^{\text {st }}$ patient, P2 is the $2^{\text {nd }}$ patient, and so forth.

\begin{tabular}{ccccccccccccc}
\hline Volume & $\begin{array}{c}\text { Motion } \\
\text { model }\end{array}$ & P1 & P2 & P3 & P4 & P5 & P6 & P7 & P8 & P9 & P10 & Ave \\
\hline V_PTV_3D & 3D & 19.1 & 17.0 & 12.2 & 12.5 & 17.0 & 21.6 & 13.8 & 5.4 & 13.8 & 23.2 & $15.6 \pm 5.2$ \\
V_PTV_4D & $4 D$ & 13.1 & 10.7 & 7.3 & 9.1 & 14.3 & 10.5 & 10.2 & 4.4 & 10.3 & 15.4 & $10.5 \pm 3.2$ \\
\hline
\end{tabular}

Table 2. The density values adopted for the override in this study. For each of the ten patients, from P1 through P10, the average, minimum, and maximum densities across ten phase-specific GTVs are provided. The minimum and maximum values were not associated with any particular phase across all patients. The densities of ITV from MIP images for each patient are also provided.

\begin{tabular}{cccccccccccc}
\hline & P1 & P2 & P3 & P4 & P5 & P6 & P7 & P8 & P9 & P10 & Ave \\
\hline Min & 0.74 & 0.57 & 0.75 & 0.60 & 0.69 & 0.64 & 0.54 & 0.63 & 0.75 & 0.67 & $0.66 \pm 0.07$ \\
Mean & 0.77 & 0.60 & 0.79 & 0.65 & 0.70 & 0.71 & 0.57 & 0.65 & 0.81 & 0.71 & $0.70 \pm 0.08$ \\
$\operatorname{Max}$ & 0.81 & 0.63 & 0.87 & 0.68 & 0.72 & 0.78 & 0.58 & 0.67 & 0.85 & 0.75 & $0.73 \pm 0.09$ \\
MIP & 0.85 & 0.62 & 0.76 & 0.68 & 0.68 & 0.78 & 0.57 & 0.63 & 0.83 & 0.76 & $0.72 \pm 0.09$ \\
\hline
\end{tabular}

AAA, 3D plans (AAA_DO_3D) were generated. All planning strategies used in this study are summarized in Table 3.

3) Planning evaluation: $4 \mathrm{D}$ dose with Acuros vs. $4 \mathrm{D}$ dose with AAA with and without DO

The plans generated by the above strategies were evaluated in terms of the $4 \mathrm{D}$ dose of the patients [15]. The setup uncertainty of GTV was considered by expanding each phase-specific GTV by a margin of $0.5 \mathrm{~cm}$, and forming a phasespecific PTV, while overriding the density of the margin area with the mean density of the corresponding GTV to model the setup uncertainty of GTV. The average PTV volumes across all patients were $10.5 \pm 3.2 \mathrm{~cm}^{3}$ with $4.4 \mathrm{~cm}^{3}$ as the minimum (for P8) and $15.4 \mathrm{~cm}^{3}$ as the maximum, as shown in Table 1 . The average density of GTV across all patients (averaged over all phases for each patient) was $0.70 \pm 0.08 \mathrm{~g} / \mathrm{cm}^{3}$ with $0.66 \mathrm{~g} / \mathrm{cm}^{3}$ as the minimum and $0.73 \mathrm{~g} / \mathrm{cm}^{3}$ as the maximum, as shown in Table 2 . The beams with their determined parameters of MUs, apertures, and locations of the 3D plan were copied to each phase-specific image and calculated with Acuros; each phase-specific plan was summed over all phases to generate $4 \mathrm{D}$ plan (AAA_4D) for each patient. The idea behind using Acuros instead of AAA was to obtain the $4 \mathrm{D}$ dose that could be delivered to the targets and neighboring organs, which then requires accurate dose calculation. The summation was performed through deformable organ/dose registration in Velocity (Varian Medical Systems, Inc), registering each phase-specific lung images, containing the associated GTV, to those at the time of maximum exhale, and therefore registering each phase-specific dose to the dose in the images at the time. The $4 \mathrm{D}$ dose calculation followed the strategy of Yoon et al. [15] which included phase-specific dose calculation and dose 
Table 3. Density override strategy adopted in this study. DO is density override.

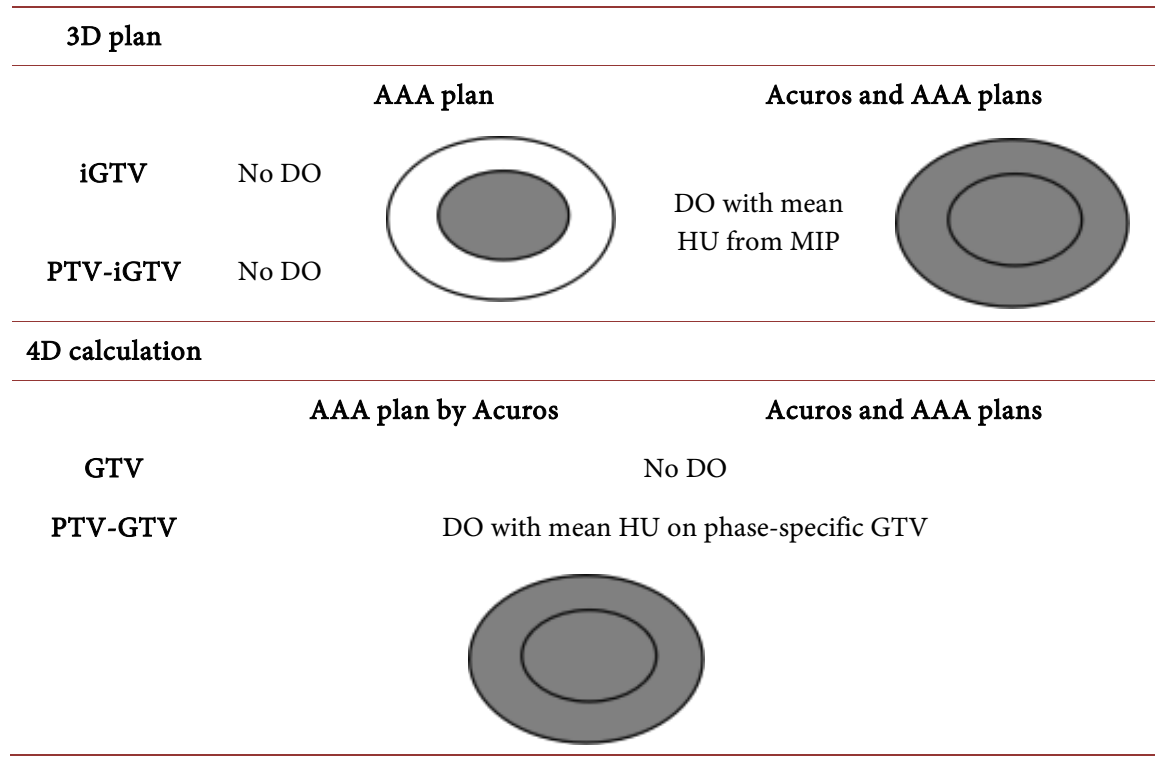

summation over phases, while the calculation utilized a Monte Carlo technique to which measurement was found comparable in accuracy. The summed dose matrix and the contour of PTV_4D (GTV at maximum exhale added by the setup margin) were transferred to the slow CT scans of each patient. The above steps were repeated to similarly acquire the $4 \mathrm{D}$ dose of the plans based on Acuros with the override (Acuros_DO_4D) and that based on AAA with the override (AAA_DO_4D). The 4D dose, Acuros_DO_4D, was compared with AAA_4D and also with AAA_DO_4D. Note that the usage of the override and Acuros was common for the case of Acuros only between the 3D and 4D doses as we intended to determine both doses accurately by using a more accurate Acuros and the geometrical modeling of the setup margins of iGTV and GTV, respectively, with the override. On the contrary, the usage was different for the case of AAA between the $3 \mathrm{D}$ and $4 \mathrm{D}$ doses as we intended to determine only the $4 \mathrm{D}$ dose accurately, provided that the 3D dose was the past clinical practice; the same was true for the case of AAA with the override, except that it was not our past clinical practice. The $4 \mathrm{D}$ doses may be interpreted as the dose that can be delivered because of the proven accuracy of Acuros as compared with measurements and Monte Carlo calculations [4] [13] and the validity of the override for the $4 \mathrm{D}$ calculations.

4) Evaluation parameters

The generated plans were evaluated in terms of average MUs for each planned beam, conformity, inhomogeneity, various dose-volume and volume-dose indices, each of which was summarized as a mean with standard deviation wherever applicable. The paired $t$ test was used to compare Acuros with AAA (without and with the override, respectively) at specific dose values, and its non-parametric analogy of the signed rank test was used for safety checking. Multiple test adjustment was not applied due to the explorative nature of this study. For all ana- 
lyses, a P value $(<0.05)$ was considered significant. Statistical analyses were performed using SAS 9.4 (SAS Institute Inc., Cary, NC).

\section{Results and Discussion}

1) Acuros with DO vs. AAA without and with DO: $3 \mathrm{D}$ and $4 \mathrm{D}$ doses in PTV.

The DVH of PTV for the case of Acuros_DO_4D, averaged over all patients, was compared with that of AAA_4D and with that of AAA_DO_4D in Figure 2. The DVHs of PTV for Acuros_DO_3D, AAA_3D, and AAA_DO_3D were also shown. The DVH of Acuros_DO_3D was found to be greater than that of AAA_3D, as shown in Figure 2, and supported by the associated P-values $(<0.05)$ in the dose range $60-79 \mathrm{~Gy}$, provided in Table 4. On the contrary, the DVH of AAA_4D was substantially greater than that of Acuros_DO_4D in the dose range 60 - $85 \mathrm{~Gy}$. This transition of the trend came from the greater MUs, averaged over the ten patients that have been determined for the AAA_3D plan than that for the Acuros_DO_3D plan, as shown in Table 5. The AAA plan had to use greater MUs to deliver to the PTV the same dose that would be delivered by the Acuros plan to the density overridden PTV. Although the calculation algorithm was different, we believe this MU difference came from the density override for the plans with Acuros that made the tumors effectively greater. This is supported by the previous findings that the use of Acuros without the density override on either case required greater MUs to deliver the same dose as calculated by using AAA in a situation where lateral scatter and buildup were not sufficiently provided such as in small lung tumors (i.e. greater dose inhomogeneity) [4] [7] [8] [9] [13]. The DVH of Acuros_DO_3D was found to be greater than that of AAA_DO_3D, as shown in Figure 2, and supported by the associated P-values $(<0.05)$ in the dose range $60-80 \mathrm{~Gy}$, provided in Table 4 . This trend was repeated for the associated $4 \mathrm{D}$ dose comparison in the range $70-80 \mathrm{~Gy}$, shown in Figure 2 and Table 4, due to greater MUs determined for Acuros_ DO_3D than those for AAA_DO_3D, shown in Table 5. The density override for Acuros provided greater MUs than that for AAA in order to deliver the

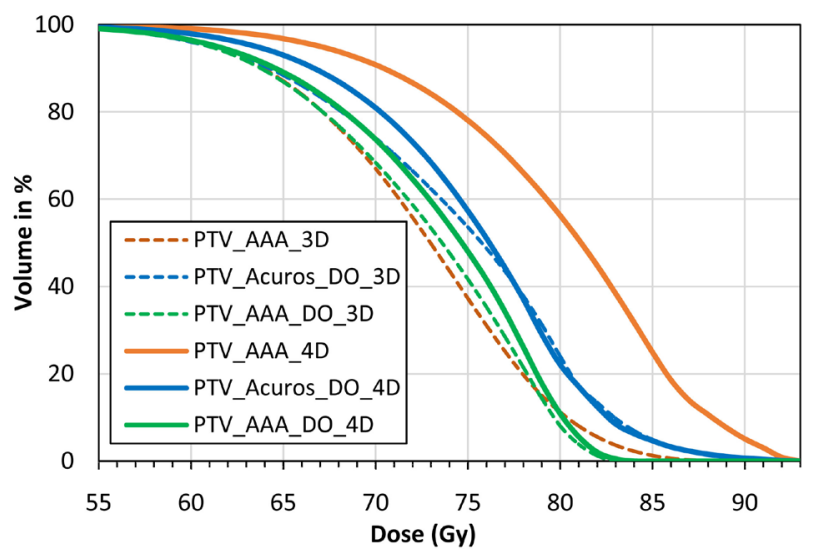

Figure 2. DVH comparison of $3 \mathrm{D}$ and $4 \mathrm{D}$ doses, averaged over the ten patients, in PTV. The $4 \mathrm{D}$ doses were all calculated by Acuros. 
Table 4. Volume (\%) coverage of dose in PTV at various dose levels for the 3D and 4D calculations, averaged over the ten patients selected for this study. ${ }^{\star}$ At $79 \mathrm{~Gy}$, the P-value is 0.0193 with the associated volume of the Acuros plan (13.3\%) which is greater than that of the AAA plan (11.2\%).

\begin{tabular}{|c|c|c|c|c|c|}
\hline Dose (Gy) & Motion account & Algorithm & Ave Vol & & alue \\
\hline \multirow{6}{*}{60} & \multirow{3}{*}{$3 \mathrm{D}$} & AAA & 95.0 & \multirow{2}{*}{0.0011 at $61 \mathrm{~Gy}$} & - \\
\hline & & Acuros_DO & 95.0 & & \multirow{2}{*}{$<0.0001$ at $61 \mathrm{~Gy}$} \\
\hline & & AAA_DO & 95.0 & - & \\
\hline & \multirow{3}{*}{$4 \mathrm{D}$} & AAA & $99.1 \pm 1.4$ & \multirow{2}{*}{0.0102} & - \\
\hline & & Acuros_DO & $97.9 \pm 1.7$ & & \\
\hline & & AAA_DO & $96.5 \pm 4.0$ & - & 0.1701 \\
\hline \multirow{6}{*}{70} & \multirow{3}{*}{$3 \mathrm{D}$} & AAA & $61.6 \pm 5.1$ & \multirow{2}{*}{$<0.0001$} & - \\
\hline & & Acuros_DO & $70.4 \pm 3.3$ & & \multirow{2}{*}{$<0.0001$} \\
\hline & & AAA_DO & $63.7 \pm 3.9$ & - & \\
\hline & \multirow{3}{*}{$4 \mathrm{D}$} & AAA & $90.8 \pm 6.3$ & \multirow{2}{*}{0.0005} & - \\
\hline & & Acuros_DO & $81.0 \pm 6.2$ & & \\
\hline & & AAA_DO & $73.7 \pm 12.7$ & - & 0.0701 \\
\hline \multirow{6}{*}{75} & \multirow{3}{*}{$3 \mathrm{D}$} & AAA & $31.1 \pm 6.0$ & \multirow{2}{*}{$<0.0001$} & - \\
\hline & & Acuros_DO & $48.8 \pm 5.6$ & & \\
\hline & & AAA_DO & $35.3 \pm 5.4$ & - & \\
\hline & \multirow{3}{*}{$4 \mathrm{D}$} & AAA & $78.1 \pm 10.7$ & \multirow{2}{*}{0.0004} & - \\
\hline & & Acuros_DO & $57.3 \pm 10.2$ & & \\
\hline & & AAA_DO & $48.0 \pm 20.2$ & - & \\
\hline \multirow{6}{*}{80} & \multirow{3}{*}{$3 \mathrm{D}$} & AAA & $8.1 \pm 4.0$ & \multirow{2}{*}{$0.0993^{*}$} & - \\
\hline & & Acuros_DO & $17.2 \pm 15.3$ & & 00075 \\
\hline & & AAA_DO & $3.9 \pm 4.5$ & - & (0) \\
\hline & \multirow{3}{*}{$4 \mathrm{D}$} & AAA & $56.3 \pm 15.2$ & \multirow{2}{*}{0.0006} & - \\
\hline & & Acuros_DO & $22.1 \pm 18.0$ & & \\
\hline & & AAA_DO & $10.9 \pm 14.3$ & - & 0.028 \\
\hline \multirow{6}{*}{85} & \multirow{3}{*}{$3 \mathrm{D}$} & AAA & $0.6 \pm 0.9$ & \multirow{2}{*}{0.2198} & - \\
\hline & & Acuros_DO & $3.4 \pm 6.5$ & & \multirow{2}{*}{0.1299} \\
\hline & & AAA_DO & 0.0 & - & \\
\hline & \multirow{3}{*}{$4 \mathrm{D}$} & AAA & $25.0 \pm 19.0$ & \multirow{2}{*}{0.0119} & - \\
\hline & & Acuros_DO & $4.7 \pm 8.5$ & & \\
\hline & & AAA_DO & 0.0 & - & 0.1170 \\
\hline & & AAA & $0.0 \pm 0.0$ & & - \\
\hline & $3 \mathrm{D}$ & Acuros_DO & $0.3 \pm 0.9$ & & \\
\hline & & AAA_DO & 0.0 & - & 0.0450 \\
\hline & & AAA & $5.1 \pm 9.0$ & & - \\
\hline & $4 \mathrm{D}$ & Acuros_DO & $0.7 \pm 2.2$ & 0.107 & \\
\hline & & AAA_DO & 0.0 & - & 0.3434 \\
\hline
\end{tabular}


Table 5. Monitor units (MUs) associated with each planned beam for the plan based on AAA, that based on Acuros with the override, and that based on AAA with the override.

\begin{tabular}{ccccc}
\hline Beam number & Algorithm & Average MU & P-value \\
\hline 1 & AAA & $871 \pm 74$ & 0.0005 & 0.0165 \\
& Acuros_DO & $821 \pm 71$ & - & - \\
AAA_DO & $795 \pm 59$ & 0.0006 & 0.0156 \\
Acuros_DO & $797 \pm 73$ & & - \\
& AAA_DO & $769 \pm 56$ & - & 0.0264 \\
& AAA & $884 \pm 100$ & 0.0012 & \\
\hline
\end{tabular}

same dose to PTV that would be delivered by AAA. This was affected by the radiation transport characteristics of Acuros that made it more accurate than AAA.

2) $4 \mathrm{D}$ vs. $3 \mathrm{D}$ doses in PTV for AAA without and with DO and Acuros with DO.

Comparing the $4 \mathrm{D}$ dose with the $3 \mathrm{D}$ dose for each of the three cases, the former tends to be greater than the latter because PTV_4D is smaller than the associated PTV_3D, thus receiving more centralized dose away from the beam penumbra while moving within the same aperture of irradiation. This geometrical argument applies to each case of AAA (although minor compared with the above MU argument), Acuros_DO, and AAA_DO. Compared with the dose difference between PTV_AAA_4D and PTV_AAA_3D for the AAA plan, the Acuros_DO plan produced a much smaller dose difference between PTV_ Acuros_DO_4D and PTV_Acuros_DO_3D. This finding was expected, as the calculation condition of the use of Acuros and the physical modeling of the density override in PTV were common between the 3D and 4D calculations. Similarly, PTV_AAA_DO_4D was found to show closer DVH to that of PTV_ AAA_DO_3D, due to the density override commonly used between them, in spite of the difference in their calculation algorithms. Wiant et al. [14] found that the density override provided a better agreement with measurements when AAA was used for planning. Although their finding may not fully apply to this study, their study shares the same application site of lung with this study. Also, as Acuros is known to offer accurate dose calculation that could provide a more inhomogeneous dose in lung tumors than the dose calculated by AAA [4] [7] [8] [9] [13], the override was further motivated in this study (additional to the arguments of setup and motion modeling). Regarding this override, this study was different from the study of Wiant et al. [14] in that it considered the 4D dose with the modeling of the override in the setup margin as a standard substituting measurement (difficult to do with the setup uncertainty modeling), while their 
study considered the measurements that could not model the presence of the target in the area of the setup margin as the standard.

3) Acuros with DO vs. AAA without and with DO: various dose indexes of PTV.

Figure 2 was evaluated in terms of various dose indexes in Table 6. The conformity index (CI) was better with the Acuros_DO plans than with the AAA plans ( 1.51 vs 1.78 for $4 \mathrm{D}$ calculation; 1.12 vs 1.21 for $3 \mathrm{D}$ ). The value of 1.78 , significantly greater than 1.51 (p-value was 0.0009 ), was well represented by the greater DVH of PTV_AAA_4D than that of PTV_Acuros_DO_4D. The index increased substantially with the $4 \mathrm{D}$ calculations from the $3 \mathrm{D}$ calculations, as the size of PTV had diminished (PTV_4D < PTV_3D), while the same apertures between the two sets of calculations (3D and 4D) were used. D99\% was smaller with the Acuros plans (58.1 vs 61.9 Gy for $4 \mathrm{D} ; 54.3$ vs 55.1 Gy for $3 \mathrm{D}$ ) that

Table 6. Dose coverage of PTV of ten patients in terms of dose evaluation indexes. CI stands for conformity index, calculated as the volume of prescribed dose (V_Dp)/ the volume of PTV (V). D99\% is the dose which $99 \%$ volume of PTV receives. D95\% is similarly defined. $\mathrm{Dp} / \mathrm{Dm}$ is the inhomogeneity index, calculated as the $\mathrm{Dp} /$ maximum dose (Dm). ${ }^{\star}$ The lowest $4 \mathrm{D}$ dose was $60 \mathrm{~Gy}$ associated with P9.

\begin{tabular}{|c|c|c|c|c|c|}
\hline Dose index & Motion account & Algorithm & Ave & & \\
\hline \multirow{6}{*}{ CI (V_PD/V) } & \multirow{3}{*}{$3 \mathrm{D}$} & AAA & $1.21 \pm 0.08$ & \multirow{2}{*}{0.0001} & - \\
\hline & & Acuros_DO & $1.12 \pm 0.06$ & & \multirow{2}{*}{0.0498} \\
\hline & & AAA_DO & $1.11 \pm 0.05$ & - & \\
\hline & \multirow{3}{*}{$4 \mathrm{D}$} & AAA & $1.78 \pm 0.37$ & \multirow{2}{*}{0.0009} & - \\
\hline & & Acuros_DO & $1.51 \pm 0.22$ & & \multirow{2}{*}{0.0069} \\
\hline & & AAA_DO & $1.39 \pm 0.27$ & - & \\
\hline \multirow{6}{*}{ D99\% } & \multirow{3}{*}{$3 \mathrm{D}$} & AAA & $55.1 \pm 0.7$ & \multirow{3}{*}{$<0.0001$} & - \\
\hline & & Acuros_DO & $54.3 \pm 0.7$ & & \multirow{2}{*}{$<0.0001$} \\
\hline & & AAA_DO & $55.0 \pm 0.6$ & & \\
\hline & \multirow{3}{*}{$4 \mathrm{D}$} & AAA & $61.9 \pm 4.6$ & \multirow{2}{*}{0.0007} & - \\
\hline & & Acuros_DO & $58.1 \pm 3.3$ & & \multirow{2}{*}{0.0092} \\
\hline & & AAA_DO & $56.4 \pm 3.8$ & - & \\
\hline \multirow{3}{*}{ D95\% } & \multirow{3}{*}{$4 \mathrm{D}$} & AAA & $67.9 \pm 4.0$ & \multirow{2}{*}{0.0007} & - \\
\hline & & Acuros_DO & $63.9 \pm 2.6^{*}$ & & \multirow{2}{*}{0.0098} \\
\hline & & AAA_DO & $62.0 \pm 3.2$ & - & \\
\hline \multirow{6}{*}{$\mathrm{Dp} / \mathrm{Dm}$} & \multirow{3}{*}{$3 \mathrm{D}$} & AAA & $0.70 \pm 0.02$ & \multirow{2}{*}{0.1964} & - \\
\hline & & Acuros_DO & $0.72 \pm 0.03$ & & \multirow{2}{*}{0.0118} \\
\hline & & AAA_DO & $0.74 \pm 0.01$ & - & \\
\hline & \multirow{3}{*}{$4 \mathrm{D}$} & AAA & $0.67 \pm 0.02$ & \multirow{2}{*}{0.0004} & - \\
\hline & & Acuros_DO & $0.71 \pm 0.03$ & & \multirow{2}{*}{0.0084} \\
\hline & & AAA_DO & $0.74 \pm 0.02$ & - & \\
\hline
\end{tabular}


provided the $4 \mathrm{D}$ dose of $58.1 \mathrm{~Gy}$, smaller than the prescribed dose (Dp) of $60 \mathrm{~Gy}$. D95\% was smaller with the Acuros plans that provided the 4D dose of $63.9 \mathrm{~Gy}$, greater than $\mathrm{Dp}$. The inhomogeneity index of $\mathrm{Dp} / \mathrm{Dm}$ was better with Acuros due to the density override in the $4 \mathrm{D}$ calculations ( 0.71 vs 0.67 ), which is visible in Figure 2 (PTV_Acuros_DO_4D vs. PTV_AAA_4D). The indices for the 3D calculations ( 0.72 vs. 0.70$)$ are not statistically distinguishable with the P-value of 0.1964, although Figure 2 shows the more inhomogeneous dose distribution in the density-overridden PTV of Acuros_DO_3D than the dose in the PTV of AAA_3D. This finding was due to the density override, because without it Acuros is known to produce greater inhomogeneous doses than AAA produces [4] [7] [8] [9] [13]. Comparing AAA_DO plans with Acuros_DO plans, the conformity index (CI) was better with the former (1.39 vs 1.51 for $4 \mathrm{D}$ calculation; 1.11 vs 1.12 for $3 \mathrm{D}$ ) with statistical significance. The value of 1.51 , greater than 1.39, was well represented by the greater DVH of PTV_Acuros_DO_4D than that of PTV_AAA_DO_4D. D99\% was smaller or greater with AAA_DO_4D ( 56.4 vs $58.1 \mathrm{~Gy}$ for $4 \mathrm{D}$; 55.0 vs $54.3 \mathrm{~Gy}$ for $3 \mathrm{D}$ ) that provided the $4 \mathrm{D}$ dose of $56.4 \mathrm{~Gy}$, smaller than Dp. D95\% was smaller with the AAA plans that provided the $4 \mathrm{D}$ dose of $62.0 \mathrm{~Gy}$ than $63.9 \mathrm{~Gy}$ with the Acuros plans. The inhomogeneity index was better with AAA ( 0.74 vs 0.71 for $4 \mathrm{D} ; 0.74$ vs 0.72 for $3 \mathrm{D}$ ) due to the characteristics of Acuros, which is somewhat visible in Figure 2 (PTV_Acuros_ DO vs PTV_AAA_DO for $4 \mathrm{D}$ and 3D, respectively; Acuros is associated with greater inhomogeneity). With the override, both algorithms predicted the $4 \mathrm{D}$ delivered dose much more accurately by the $3 \mathrm{D}$ planned dose than the AAA algorithm did without the override. However, the override for Acuros provided a greater value of D99\% than that for AAA ( 58.1 vs $56.4 \mathrm{~Gy}$ ) due to the greater MUs calculated for Acuros, as shown in Table 5.

4) Acuros with DO vs. AAA without and with DO: various dose indexes of GTV.

The DVH of GTV at maximum exhale, averaged over all patients, for Acuros_DO_4D was compared with AAA_4D and AAA_DO_4D in Figure 3. The

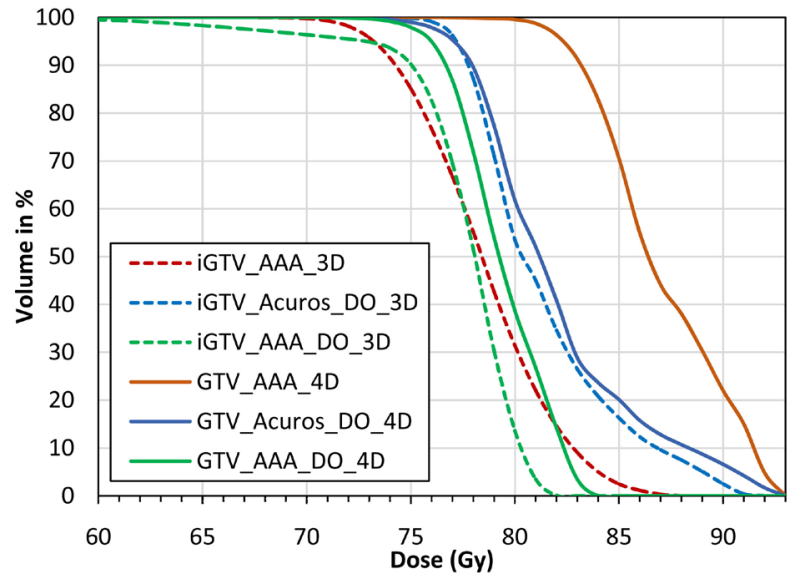

Figure 3. DVH comparison of 3D and $4 \mathrm{D}$ doses, averaged over the ten patients, in iGTV and GTV. The 4D doses were all calculated by Acuros. 
DVHs of iGTV for the cases of Acuros_DO_3D, AAA_3D, and AAA_DO_3D are also shown. Table 7 lists volume data at selected dose levels in Figure 3. Comparing the 3D calculations, the DVH of Acuros_DO_3D was found to be greater than that of AAA_3D, as supported by the associated P-values $(<0.05)$ in the dose range 71 - $79 \mathrm{~Gy}$, provided in Table 7. Although the DVH comparison clearly showed the similar amount of differences between the two in the dose range beyond $79 \mathrm{~Gy}$, the associated P-values $(>0.05)$ did not reach statistical significance, affected by zero values of \% volume for both Acuros and AAA calculations for multiple patients. Similar to the finding as shown in Figure 2, the DVH of AAA_4D was substantially greater than that of Acuros_DO_4D in the

Table 7. Volume (\%) coverage of dose in iGTV and GTV of the ten patients. ${ }^{*}$ From the dose of 71 to $79 \mathrm{~Gy}$, the values were smaller than 0.05 . ${ }^{\circledR}$ At $88 \mathrm{~Gy}$, the value was 0.044 . D100\% was 77, 72, and 71 Gy for AAA_4D, Acuros_DO_4D and AAA_DO_4D, respectively.

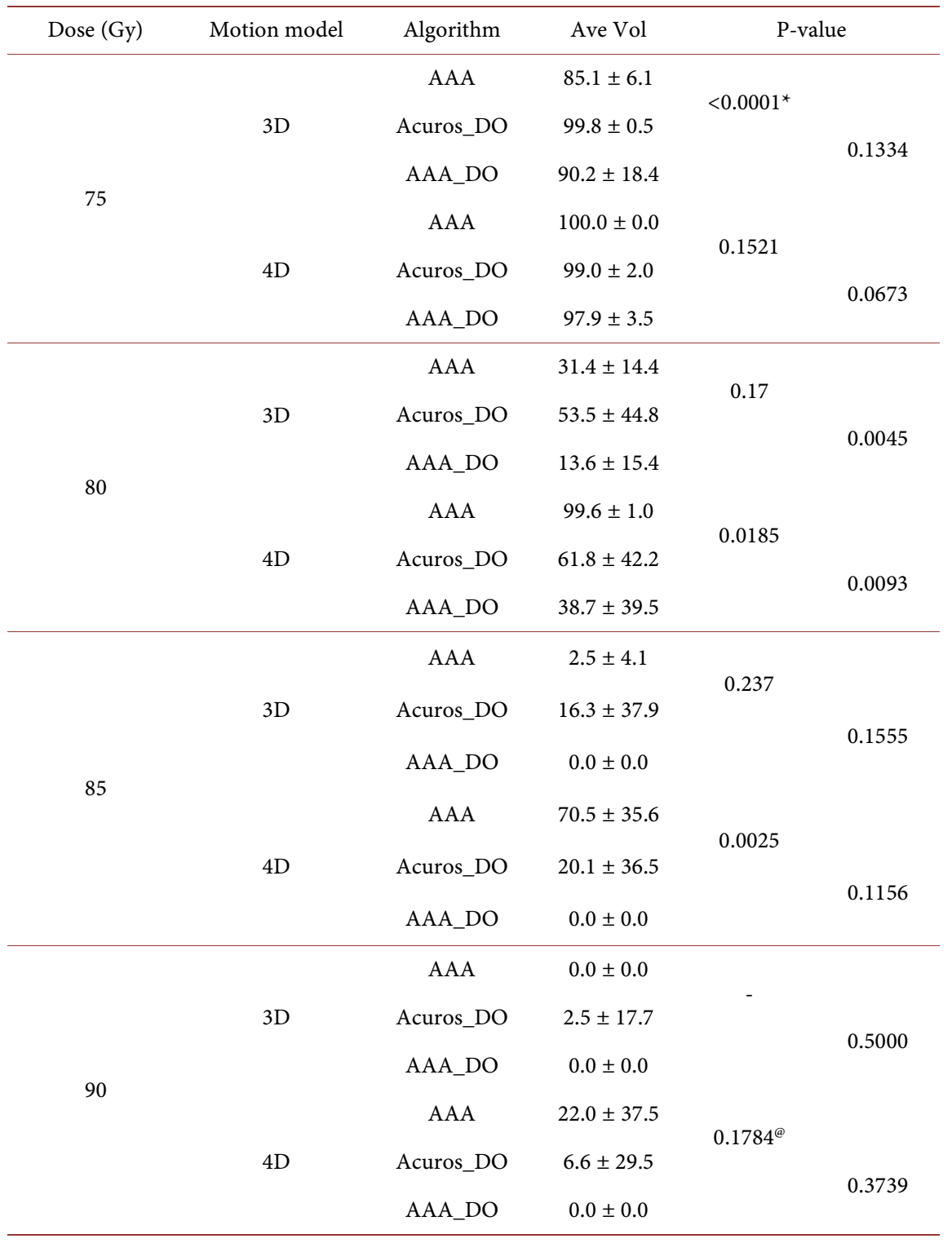


dose range from 80 to $88 \mathrm{~Gy}$, as supported by the associated P-values. Comparing Figure 3 with Figure 2 and Table 7 with Table 4, the greater values of the doses were deliverable to GTV than those to PTV. This came from the more central and greater dose GTV receives than that PTV receives. The greater dose was due to the significant dose inhomogeneity provided in PTV (i.e. small targets) with its maximum dose placed in GTV. As a result, GTV received a substantially greater dose than $\mathrm{Dp}$ (prescribed to the PTV of the 3D calculations) for both 3D and 4D calculations. The 4D dose to GTV in Figure 3 was 72 and 77 Gy (D100\%) for the cases of Acuros and AAA, respectively. Comparing the plan of Acuros_DO with that of AAA_DO, the DVH of iGTV_Acuros_DO_3D was found to be greater than that of iGTV_AAA_DO_3D, as shown in Figure 3, due to the greater dose inhomogeneity of Acuros that associates the greater dose to the central region of PTV where iGTV is located. The DVH of GTV_Acuros_ DO_4D was substantially greater than that of GTV_AAA_DO_4D, as shown in Figure 3 and Table 7, due to the greater MUs for the former, as shown in Table 5.

5) Acuros with DO vs. AAA without and with DO: $4 \mathrm{D}$ doses in lung and CW.

The DVHs of lungs and CW, averaged over all patients, for Acuros_DO_4D were compared with those of AAA_4D and AAA_DO_4D in Figure 4. Table 8 showed the data in Figure 4 at selected dose values for lungs (i.e. lungs-GTV)

Table 8. Volume coverage of $4 \mathrm{D}$ dose to lungs and $\mathrm{CW}$ of the ten patients.

\begin{tabular}{|c|c|c|c|c|}
\hline Dose (Gy) & Algorithm & Ave lung Vol (\%) & \multicolumn{2}{|c|}{$\mathrm{P}$-value } \\
\hline \multirow{3}{*}{10} & AAA & $8.08 \pm 2.65$ & 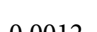 & \multirow{3}{*}{0.4779} \\
\hline & Acuros_DO & $7.70 \pm 2.54$ & & \\
\hline & AAA_DO & $7.65 \pm 2.61$ & & \\
\hline \multirow{3}{*}{20} & AAA & $4.04 \pm 1.67$ & \multirow{3}{*}{0.0006} & \\
\hline & Acuros_DO & $3.71 \pm 1.61$ & & \\
\hline & AAA_DO & $3.65 \pm 1.63$ & & \\
\hline \multirow{3}{*}{30} & AAA & $2.26 \pm 1.17$ & \multirow{3}{*}{0.0004} & \\
\hline & Acuros_DO & $2.05 \pm 1.10$ & & \\
\hline & AAA_DO & $2.00 \pm 1.10$ & & \\
\hline \multirow{3}{*}{40} & AAA & $1.36 \pm 0.77$ & \multirow{3}{*}{0.0017} & \\
\hline & Acuros_DO & $1.20 \pm 0.68$ & & 00241 \\
\hline & AAA_DO & $1.16 \pm 0.67$ & & \\
\hline \multirow{3}{*}{60} & AAA & $0.49 \pm 0.21$ & \multirow{3}{*}{0.0016} & \multirow{3}{*}{0.0067} \\
\hline & Acuros_DO & $0.40 \pm 0.17$ & & \\
\hline & AAA_DO & $0.37 \pm 0.16$ & & \\
\hline Vol & Algorithm & Ave $C W$ vol $(\mathrm{mL})$ & \multirow{4}{*}{0.0136} & \\
\hline \multirow{3}{*}{ V30 Gy } & AAA & $10.18 \pm 10.16$ & & \multirow{3}{*}{0.0155} \\
\hline & Acuros_DO & $7.87 \pm 8.26$ & & \\
\hline & AAA_DO & $6.99 \pm 8.17$ & & \\
\hline
\end{tabular}




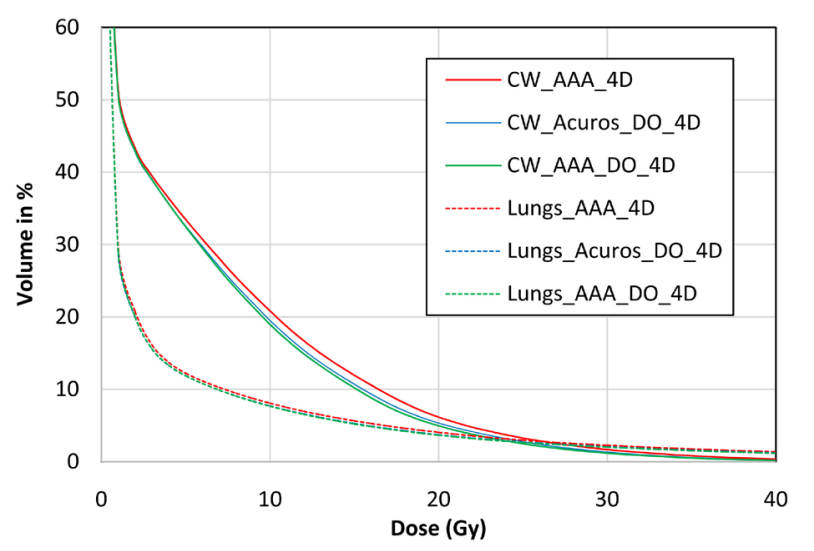

Figure 4. DVH comparison of $4 \mathrm{D}$ doses, averaged over the ten patients, in $\mathrm{CW}$ and lungs (lungs-GTV). The 4D doses were all calculated by Acuros.

and CW. At all dose levels from $10 \mathrm{~Gy}$, the Acuros_DO_4D plans provided each $4 \mathrm{D}$ dose to smaller lung volumes than the volumes the AAA_4D plans provided, although the volume saving was minor ( $7.7 \%$ vs $8.1 \%$, and so forth). This advantage came from the smaller MUs associated with the Acuros_3D plans than those with the AAA_3D plan, as described above. At the doses above $40 \mathrm{~Gy}$, with the density override the Acuros_DO_4D plans provided each 4D dose to greater lung volumes than those the AAA_DO_4D provided to $(1.20 \%$ vs $1.16 \%$, and so forth), although the difference is very small, due to the greater MUs associated with the former. Similarly, the Acuros_DO_4D plans provided the dose of $30 \mathrm{~Gy}$ to a smaller volume of CW than that of the AAA_4D plans (7.87 vs $10.18 \mathrm{~mL}$ ). Also, the Acuros_DO_4D plans provided the dose of $30 \mathrm{~Gy}$ to a greater volume of CW than that of the AAA_DO_4D plans (7.87 vs $6.99 \mathrm{~mL}$ ).

\section{Further Discussion}

The goal of planning on an expanded target by the setup margin (namely, PTV) is to model accurately the dose that can be deliverable in the target (iGTV or GTV) which occupies the setup margin during treatments. This can be done with reasonable accuracy if the following conditions are met: 1) The setup margin is of the similar density to the target's density in terms of similarity in radiation transport and dose deposition (namely, density effect); 2) The geometrical modeling of the location of the target is done correctly, based on the nature of the setup margin, in terms of its distance to beam penumbra (distance effect). Note that the distance from the PTV boundary to the aperture boundary is different from the distance from the target boundary (with positional setup uncertainty) to the latter; 3) The target is relatively large (size effect), so that sufficient dose buildup and lateral scatter provide a flat homogeneous dose in it and the fraction of the target volume that receives penumbral dose is relatively small compared with the entire volume. Therefore, DVH in the target is similar to that in its PTV. In lung SBRT, none of the above three conditions is met. To make matters worse, its small tumor is surrounded by lung of a lesser density, causing 
the radiation beam to pass through the lung before it hits the tumor, and further increasing dose inhomogeneity in the target (buildup effect) from the already inhomogeneous dose in it.

In this study, the density override was intended to correctly account for the density effect and the distance effect (in part). This approach, however, counteraffected the dose calculation in terms of the size and buildup effects, as it increased the effective size of the target (from GTV to PTV). As a result, dose in PTV_4D could not accurately represent doses in iGTV_4D, and therefore GTV_4D. The impact of the two effects on the 4D dose evaluation could be quantitatively estimated using the case of $\mathrm{P} 9$ that exhibited the lowest $4 \mathrm{D}$ dose of 60 Gy in D95\% of PTV among the ten patients. We reasoned that the effects would be greatest when the $4 \mathrm{D}$ dose is smallest, affected most greatly by the buildup and scatter characteristics. For this case, when the density override was not performed, the $4 \mathrm{D}$ dose of D95\% was determined to be 58.2 Gy with the decreased amount of 1.8 Gy $(-3.0 \%)$. While this evaluation was done using the image sets of all phases, a similar evaluation was performed on the image set at the mid inhale only. Compared with the former, the latter could provide us with the impact of the two effects more accurately in principle because it involved the GTV that was most centrally placed to the aperture of irradiation among the GTVs of all phases, and therefore least affected by beam penumbrae. From the latter, D95\% was found to be $65.4 \mathrm{~Gy}$ without the override and $68.8 \mathrm{~Gy}$ with the override. Provided that GTV can assume any position within the boundary of the associated PTV, the difference of $3.4 \mathrm{~Gy}(+5.7 \%)$ could be a maximum estimation of the impact of the two effects. Therefore, it is possible that the $4 \mathrm{D}$ dose values were overestimated in this study by a few Gy as a maximum.

For further study, a similar study may be pursued for tumors with different sizes $(<2 \mathrm{~cm}$ or $>4 \mathrm{~cm}$ ), because the size variation affects the dose buildup and lateral scatter of Acuros as well as AAA and for the intensity modulated treatment of more static tumors.

\section{Conclusion}

Compared with the AAA-based planning, the Acuros-based planning with the density override provided a better agreement between the $3 \mathrm{D}$ and $4 \mathrm{D}$ plans, the former being a good predictor of the 4D dose. Regarding the 4D dose, Acuros provided better dose conformity and inhomogeneity, thereby saving more lung tissues and neighboring organs; it provided the greater volume at Dp than the prescribed (97.9/95\%), although smaller than that by the AAA-based plan (99.1\%). The Acuros-based planning with the override was determined to be more accurate. The AAA-based planning with the override provided similar findings to those of the Acuros-based planning with the override. However, it provided less 4D dose than that of the latter in PTV (D95\%: 62 vs $63.9 \mathrm{~Gy}$ ), making the latter a more conservative planning technique. Considering the possible overestimation of $4 \mathrm{D}$ dose, due to the effect of the size and buildup in- 
crease described in the discussion section, the Acuros-based planning with the override is recommended clinically.

\section{Conflicts of Interest}

The authors declare no conflicts of interest regarding the publication of this paper.

\section{References}

[1] Timmerman, R., Paulus, R., Galvin, J., et al. (2010) Stereotactic Body Radiation Therapy for Inoperable Early Stage Lung Cancer. JAMA, 303, 1070-1076. https://doi.org/10.1001/jama.2010.261

[2] Bezjak, A. (2011) RTOG0813 Seamless Phase I/II Study of SBRT for Early Stage, Centrally Located, Non-Small Cell Lung Cancer in Medially Inoperable Patients. Radiation Therapy Oncology Group, USA.

[3] Rana, S., Rogers, K., Pokharel, S. and Cheng, C. (2014) Evaluation of Acuros XB Algorithm Based on RTOG 0813 Dosimetric Criteria for SBRT Lung Treatment with RapidArc. Journal of Applied Clinical Medical Physics, 15, 118-129. https://doi.org/10.1120/jacmp.v15i1.4474

[4] Shiraishi, S., Santos, L., Antolak, J., et al. (2019) Phantom Verification of AAA and Acuros Dose Calculations for Lung Cancer: Do Tumor Size and Regression Matter? Practical Radiation Oncology, 9, 29-37. https://doi.org/10.1016/j.prro.2018.06.008

[5] Peterlin, P., Stanic, K., Mendez, I., et al. (2017) Treating Lung Cancer with Dynamic Conformal Arc Therapy: A Dosimetric Study. Radiation Oncology, 12, Article No. 93. https://doi.org/10.1186/s13014-017-0823-y

[6] Ross, C., Kim, J., Chen, Z., et al. (2011) A Novel Modified Dynamic Conformal Arc Technique for Treatment of Peripheral Lung Tumors Using Stereotactic Body Radiation Therapy. Practical Radiation Oncology, 1, 126-134.

[7] Tsuruta, Y., Nakata, M., Nakamura, M., et al. (2014) Dosimetric Comparison of Acuros XB, AAA, and XVMC in Stereotactic Body Radiotherapy for Lung Cancer. Medical Physics, 41, Article ID: 081715. https://doi.org/10.1118/1.4890592

[8] Liu, H., Nugent, Z., Clayton, R., et al. (2014) Clinical Impact of Using the Deterministic Patient Dose Calculation Algorithm Acuros XB for Lung Stereotactic Body Radiation Therapy. Acta Oncologica, 53, 324-329. https://doi.org/10.3109/0284186X.2013.822552

[9] Ong, C., Ang, K., Soh, R., et al. (2017) Dosimetric Comparison of Peripheral NSCLC SBRT Using Acuros XB and AAA Calculation Algorithms. Medical Dosimetry, 42, 216-222. https://doi.org/10.1016/j.meddos.2017.05.005

[10] Huang, B., Wu, L., Lin, P., et al. (2015) Dose Calculation of Acuros XB and Anisotropic Analytic Algorithm in Lung Stereotactic Body Radiotherapy Treatment with Flattening Filter Free Beams and the Potential Role of Calculation Grid Size. Radiation Oncology, 10, Article No. 53. https://doi.org/10.1186/s13014-015-0357-0

[11] Kroon, P., Hol, S. and Essers, M. (2013) Dosimetric Accuracy and Clinical Quality of Acuros XB and AAA Dose Calculation Algorithm for Stereotactic and Conventional Lung Volumetric Modulated Arc Therapy Plans. Radiation Oncology, 8, Article No.149. https://doi.org/10.1186/1748-717X-8-149

[12] Yan, C., Combine, A. and Bednarz, G. (2017) Clinical Implementation and Evaluation of the Acuros Dose Calculation Algorithm. Journal of Applied Clinical Medical Physics, 18, 195-209. https://doi.org/10.1002/acm2.12149 
[13] Bush, K., Gagne, I.M., Zavgorodni, S., et al. (2011) Dosimetric Validation of Acuros SB with Monte Carlo Methods for Photon Dose Calculations. Medical Physics, 38, 2208-2221. https://doi.org/10.1118/1.3567146

[14] Wiant, D., Vanderstraeten, C., Maurer, J., et al. (2014) On the Validity of Density Overrides for VMAT Lung SBRT Planning. Medical Physics, 41, Article ID: 081707. https://doi.org/10.1118/1.4887778

[15] Yoon, J., Jung, J., Kim, J., et al. (2016) Four-Dimensional Dose Reconstruction Through in vivo Phase Matching of Cine Images of Electronic Portal Imaging Device. Medical Physics, 43, 4420-4430. https://doi.org/10.1118/1.4954317

[16] Takao, S., Miyamoto, N., Matsuura, T., et al. (2016) Intrafractional Baseline Shift or Drift of Lung Tumor Motion During Gated Radiation Therapy with a Real-Time Tumor-Tracking System. International Journal of Radiation Oncology, Biology, Physics, 94, 172-180. https://doi.org/10.1016/j.ijrobp.2015.09.024

[17] Ma, J., Liu, Y., Sun, L., et al. (2018) Chest Wall Toxicity after Stereotactic Body Radiation Therapy: A Pooled Analysis of 57 Studies. International Journal of Radiation Oncology, Biology, Physics, 103, 843-850.

https://doi.org/10.1016/j.ijrobp.2018.11.036 